\title{
NOTES ON ABSTRACT DISTANCE GEOMETRY I. THE ALGEBRAIC DESCRIPTION OF GROUND SPACES.*
}

\author{
DAVid ElLis
}

(Received December 8, 1951)

1. Introduction. In this note we employ the terminology of our foundational paper ${ }^{1)}$ on geometry in abstract distance spaces. For the sake of completeness, however, the terms used in the note are defined below. We assume a familiarity on the part of the reader with the terminology of single operation algebra. The purpose of the note is to correlate certain distance-theoretic properties of a ground space with algebraic properties of its metroid (defined below).

A set $G$ is called a ground space if there is a function $d(x, y)$, called the distance function of $G$, on $G G$ to $G$. This distance function may also be regarded as the composition of a groupoid on the set $G$. This groupoid is called the metroid of the ground space. We denote it by $M(G)$ or simply by $\boldsymbol{M}$. When $\boldsymbol{M}(G)$ is under consideration, we also write $x y$ for $d(x, y)$ since the former is more suggestive of an algebraic operation. Thus, we have a set and a binary operation table which may be regarded either as the distance table for the ground space $G$ or as the Cayley square for $M$.

A ground space $G$ may satisfy one or both of the following distancetheoretic conditions :

Symmetry: $d(x, y)=d(y, x)$ for all $x, y \in G$.

Vanishing: $d(x, x)=d(y, y) \neq d(x, y)$ for all $x, y \in G$

with $x \neq y$. If $G$ satisfies the vanishing condition we denote by 0 the element $d(x, x)$,

A ground space which satisfies the conditions of symmetry and vanishing is called generalized semimetric.

A generalized semimetric ground space is called normal if it satisfies the condition:

Normality: $d(x, 0)=x$ for all $x \in G$.

When we speak of normal ground space it is to be understood that the ground space under consideration is required to be generalized semimetric.

A ground space $G$ is called basal if each element of $G$ forms a complete metric base for $G$; that is, If $p \in G$ then for any $d \in G$ there is a unique $q \in G$ with $d(p, q)=d$.

A generalized semimetric ground space has the property of triangular

\footnotetext{
* Presented to the American Mathematical Society; Christmas, 1950.

1) DAVID ELlIS, Geometry in abstract distance spaces, to appear in Publicationes Mathematicae (Debrecen), Vol. 2 (1950), Issue 1.
} 
fixity if $d(x, d(x, y))=y$ for all $x, y \in G$. Again, when speaking of triangular fixity it is to be understood that the ground space under consideration is generalized semimetric.

We say that a groupoid with unit element 0 is nilpotent (of index 2) provided $x x=0$ for all $x \in G$. Note that this is distinct from the use of the term by some writers on algebra.

2. The algebraic description of ground spaces. In all the following $G$ is an arbitrary ground space and $M$ is its metroid. The proofs of the Theorems 1,2, 3 and Lemmas $2.1,2.2$, and 7.1 are all quite easy and are all straightforward verification except in Lemma 7.1 where it is merely necessary to take two elements identical in the associative law.

Theorem 1. $G$ is basal if and oniy if $M$ is a quasigroup.

LEMMA 2.1. G satisfies the symmetry condition if and only if $M$ is commutative.

LEMMA 2.2. G satisfies the vanishing condition if and only if $M$ has an element 0 with respect to which it is nilpotent (not necessarily a unit element) and the coset of $M-(x)$ by $x$ does not contain 0 for any $x$.

THEOREM 2. $G$ is generalized semimetric if and only if $M$ is a commutative groupoid nilpotent with respect to one of its elements (not necessarily a unit element) and the coset of $M-(x)$ by $x$ does not contain this element for any $x$.

THEOREM 3. $G$ is a normal ground space if and only if $M$ is a commutative groupoid with a unit element with respect to which it is nilpotent and the coset of $M-(x)$ by $x$ does not contain the unit element for any $x$.

LEMма 7.1. If $G$ is normal and $M$ is a semigroup then $G$ has the property of triangular fixity.

From the preceding Lemmas and Theorems we may deduce the following theorems giving distance-theoretic properties of a ground space implied by the corresponding metroid being of a standard type.

THEOREM 4. A ground space whose metroid is a commutative groupoid satisfies the condition of symmetry.

THEOREM 5. A ground space whose metroid is a quasigroup is basal.

THEOREM 6. A ground space whose metroid is a commutative nilpotent loop is normal and basal.

THEOREM 7. A ground space whose metroid is a nilpotent Abelian group is normal, basal, and triangularly fixed (has the property of triangular fixity).

3. Two examples. As examples to illustrate the preceding section we consider $\mathfrak{R}^{*}$, the non-negative real ray with Euclidean distance, and $\mathfrak{B}$, any autometrized Boolean algebra ${ }^{2)}$. We know that the metroid of $\mathfrak{B}$ is

2) DAVID Ell.IS, Autometrized Boolean algebras I, to appear in Canadian Journal of Mathematics. 
the additive group of the Boolean ring associated with $\mathfrak{B}$ (a reference to this result which is an immediate consequence of a theorem of Stone is given in the paper of footnote 2). Hence, the metroid of $\mathfrak{B}$ is a nilpotent Abelian group and Theorem 7 yields that $\mathfrak{B}$ is normal, basal, and triangularly fixed. These properties of $\mathfrak{B}$ were demonstrated independently of the present observations in the paper of footnote 2 .

While $\Re^{*}$, the prototype of ground spaces, is normal, it is easily seen that it is not basal nor triangularly fixed. Hence, from Theorem 6 and Lemma 7.1 we have that the metroid of $\mathfrak{R}^{*}$ (namely, the set of non-negative reals with composition $|x-y|)$ is neither a loop nor a semigroup.

4. Proposed problems. The preceding note raises some questions unanswered in it. The most interesting are:

1. What distance-theoretic property together with triangular fixity im. plies (non-trivially) that the associated metroid is a semigroup?

2. It seems probable that considerable agebraic interest may attach to the metroids corresponding to ground spaces in which every $n$-distinct elements form a complete metric base but which are not necessarily basal. Such a groupoid might be called an $n$-quasigroup and defined: A groupoid $G$ is an $n$-quasigroup if each of the two systems

$$
\begin{aligned}
& a_{i} x=b_{i} ; i=1,2, \cdots, n \\
& y a_{i}=b_{i} ; i=1,2, \cdots, n
\end{aligned}
$$

of equations have a unique solution when $\left(a_{1}, \cdots, a_{n}\right)$ and $\left(b_{1}, \cdots, b_{n}\right)$ are any two $n$-tuples each consisting of $n$ distinct elements of $G$. These would be generalizations of the notion of quasigroup in that a 1-quasigroup is a quasigroup proper.

THE UNIVERSITY OF FLORIDA 